\title{
Propensity score matching analysis on inpatient period differences of hemorrhagic stroke survivors depending on medical insurance coverage
}

\author{
Sang-Mi Kim ${ }^{\mathrm{a}}$, Young Kim ${ }^{\mathrm{b}}$, Seong-A Lee ${ }^{\mathrm{c}}$ \\ ${ }^{a}$ Department of Business Administration, Graduate School of Business, Ewha Womans University, Seoul, Republic of Korea \\ ${ }^{b}$ Wellness Coaching Service Research Center, Soonchunhyang University, Asan, Republic of Korea \\ 'Department of Occupational Therapy, College of Medical Science, Soonchunhyang University, Asan, Republic of Korea
}

Objective: The purpose of this study was to compare the differences in the length of hospital stay between hemorrhage stroke survivors with health insurance and those with medical care after controlling all factors except for the type of medical insurance by using the propensity score matching (PSM) method.

Design: Retrospective cohort study.

Methods: Data from the Korean National Centers for Disease Control and Prevention's In-Depth Discharge Injury Survey between the years 2006 and 2012 were used for analysis. A total of 4,538 cases were defined as persons with hemorrhagic stroke (I60-I62) based on the block of categories in the International Classification of Diseases (10th). In order to analyze the inpatient period differences depending on the type of health care, which reflects one's socio-economic level, the chi-square and t-test was conducted.

Results: Frequency and percentage were presented, and regression analysis was used to determine the factors affecting the inpatient period. Age, severity of disease, treatment outcome, and post-discharge status were no longer statistically significant after matching. The inpatient period of the persons receiving medical aid benefits was found to be significantly longer than those with national health insurance $(p<0.05)$.

Conclusions: The factors influencing the inpatient period of hemorrhagic stroke survivors were treatment outcomes, severity of disease, hospital admission process, and the type of health care. It is necessary for systematic and comprehensive governmental management for persons with hemorrhagic stroke to be transferred to long-term care facilities.

Key Words: Delivery of health care, Inpatient, National health insurance, Stroke

\section{Introduction}

There is a high likelihood that long-term hospitalization will increase sharply due to the rapid increase in the elderly population, the fee-for-service system, and the absence of primary care system to manage preventable hospitalizations [1]. Those receiving medical aid benefits, which accounts for $2.8 \%$ of the total population, are older and have greater disease severity than those with national health insurance
[2]. Despite the surging medical costs of the patients receiving medical aid, the medical care budget is insufficient, thus leading to prolonged financial losses [3].

According to the National Statistical Office, the common causes of death in Korea is malignant neoplasm, heart disease, and cerebrovascular disease. Cerebrovascular disease was the second most common cause of death in Korea, following malignant neoplasms (60.2 per 100,000 population) in 2017 [4]. Stroke, the second leading cause of death, is a

Received: 19 March, 2019 Revised: 10 May, 2019 Accepted: 27 May, 2019

Corresponding author: Seong-A Lee (ORCID https://orcid.org/0000-0001-6263-9916)

Department of Occupational Therapy, College of Medical Science, Soonchunhyang University, 22 Soonchunhyang-ro, Asan 31538, Republic of Korea Tel: 82-41-530-3037 Fax: 82-41-530-3085 E-mail: myanmy@sch.ac.kr

(c) This is an Open-Access article distributed under the terms of the Creative Commons Attribution Non-Commercial License (http://creativecommons.org/licenses/ by-nc/4.0) which permits unrestricted non-commercial use, distribution, and reproduction in any medium, provided the original work is properly cited.

Copyright $\odot 2019$ Korean Academy of Physical Therapy Rehabilitation Science 
disease that increases the burden of medical expenses due to the occurrence of severe disability and complications. Of the medical expenses, the one-year expenditure from the date of illness accounted for $59 \%$ of admission and $13 \%$ of outpatient services [3]. Even if the stroke does not lead to death, it causes serious neurological disabilities and high cost of treatment and rehabilitation. According to the OECD's 'Health at a Glance 2015', the average length of hospital stay in Korea is 16.5 days, more than twice the OECD average of 8.1 days [1]. The average duration of hospitalization for hemorrhagic stroke survivors in Korea is reported to be between 28.9 to 37.85 days, which is much longer than that of 8.4 days in the US $[5,6]$. The mean duration of hospitalization for hemorrhagic stroke is 32-37 days, which is longer than that of ischemic stroke (17-19 days) [7,8]. In addition, the total annual socioeconomic cost of medical users due to hemorrhagic stroke accounted for $35.4 \%$ of the total socioeconomic costs (I60-I69, G45), combining both the outpatient and inpatient medical users [9].

Due to the increasing length of hospital stay, the need for studies on the use of medical facilities by the persons with medical aid is emphasized, and a variety of studies have been conducted $[6,10,11]$. However, existing studies have limitations of not compensating for the differences in the prevalence and severity of the patients with national health insurance and medical aid [12,13].

Therefore, the purpose of this study was to compare the inpatient periods of hemorrhagic stroke survivors with national health insurance and medical aid by using the propensity score matching (PSM) analysis. Unlike previous studies that did not consider the patient and the disease characteristics, this study controlled all confounding factors other than the type of health care and equalized the subject characteristics in the two groups by the PSM. The presence of differences in the general characteristics of the patients with national health insurance and those receiving medical aid were first examined. The characteristics of the two groups were then analyzed after controlling the confounding factors. Lastly, the factors affecting the inpatient period of hemorrhagic stroke survivors were analyzed.

\section{Methods}

\section{Subjects}

This study used data from the Korean National Hospital Discharge In-depth Injury Survey conducted from 2006 to 2012 by the National Centers for Disease Control and
Prevention. Soonchunhyang Institutional Review Board certified center approved the this research (SCH IRB No. 2016-BM-036-02). Included subjects were defined as persons with hemorrhagic stroke (coded I60-I62) with the final diagnosis at discharge according to the categorical blocks of the ICD-10, which is based on the detailed criteria and evaluation index of the acute stroke evaluation of the Health Insurance Evaluation and Assessment Agency [14,15]. The size of the medical institutions was limited to 500 to 999 beds because duration of hospitalization can differ according to the size of medical institutions [14]. Out of the 4,904 selected cases, a total of 4,538 cases were analyzed excluding those under 18 years of age, with workers' compensation or automobile insurance, with unknown admission route, and with an inpatient period exceeding 365 days.

\section{Data collection}

In this study, the length of hospital stay (inpatient period) was defined as use of medical service among those affected by hemorrhagic stroke. It was simply calculated by subtracting the discharge date from the admission date. Only those with less than 365 days of hospitalization were included for analysis.

Patient characteristics were classified into sex, age, place of residency, severity of disease, hospital admission process, treatment outcome, and post-discharge status. Age refers to the age at the time of admission, and when the patient and medical institution were from the same administrative district, they were marked as 'congruent'. Severity of disease was calculated by using the Charlson Comorbidity Index (CCI). The CCI is the most widely used method for severity correction; it determines any comorbidity that affects treatment outcomes and corrects the severity by using the weight of comorbidity or the presence of disease [16-18]. Hospital admission process was classified into emergency and outpatient, and the treatment results were classified as improved and not improved. Post-discharge status was classified into returned home, transferred, and deceased. The characteristics of the medical institutions were selected based on the place of location; capital city, metropolitan city, and provincial area.

\section{Data analysis}

Propensity score matching is a method of matching up similar subjects based on the propensity score in situations in which covariates are controlled in the laboratory and in the control of the covariates of the control group. Therefore, 
because the selection bias is reduced and the subjects are reconstructed and compared, there is an advantage in controlling the confounding by comparing the differences between comparison groups more accurately. The logistic regression analysis of variables, such as sex, age, residence, Charlsonindex, hospital assay, treatment exit, post-discahridge, and hospital area found to affect the number of days of funding were estimated. The next estimated values were compared to match a group with a similar propensity score as the control. This was considered as near-neighbor matching, in which 296 people were matched according to health insurance and medical benefits. Finally, it was verified that the covariates represent the end distribution of covariates in the experimental group (national health insurance) and the control group (medical benefits). To investigate the distribution of the general characteristics of the study subjects, which were those with national health insurance and medical aid, the frequency and percentage were indicated by descriptive statistics. The chi-square and t-test were used to determine whether there was a statistically significant difference between patient characteristics and medical institution characteristics. Next, the PSM was used to effectively control the factors that could affect the inpatient period other than the type of health care.

Regression analysis was conducted to reveal the factors affecting the inpatient period after matching the characteristics of patients with national health insurance and medical aid by using the PSM. Data collected in this study were analyzed by STATA 12.0, and the significance level was set at $5 \%(p<0.05)$.

\section{Results}

\section{General characteristics}

Among the patient characteristics, age, Charlson severity level, treatment outcome, and post-discharge status showed

Table 1. General characteristics before matching

$(\mathrm{N}=4,538)$

\begin{tabular}{|c|c|c|c|}
\hline Characteristic & National health insurance $(n=4,242)$ & Medical aid $(n=296)$ & $\chi^{2 / t}(p)$ \\
\hline \multicolumn{4}{|l|}{ Sex } \\
\hline Male & $2,036(48.0)$ & $127(42.9)$ & $2.870(0.090)$ \\
\hline Female & $2,206(52.0)$ & $169(57.1)$ & \\
\hline Age (y) & $59.17(14.19)$ & $63.03(14.81)$ & $-4.360(<0.001)$ \\
\hline \multicolumn{4}{|l|}{ Residence } \\
\hline Consistency & $3,163(74.6)$ & $210(71.0)$ & $1.900(0.168)$ \\
\hline Inconsistency & $1,079(25.4)$ & $86(29.1)$ & \\
\hline \multicolumn{4}{|l|}{ Charlson Index } \\
\hline CCI 0 & 2,287 (53.9) & $128(43.2)$ & $16.270(0.001)$ \\
\hline CCI 1 & $1,224(28.9)$ & $99(33.5)$ & \\
\hline CCI 2 & $393(9.3)$ & $31(10.5)$ & \\
\hline CCI 3+ & $338(8.0)$ & $38(12.8)$ & \\
\hline \multicolumn{4}{|l|}{ Hospital admission } \\
\hline Emergency & $3,637(85.7)$ & $262(88.5)$ & $1.760(0.184)$ \\
\hline Outpatient & $605(14.3)$ & $34(11.5)$ & \\
\hline \multicolumn{4}{|l|}{ Treatment outcome } \\
\hline Improved & $3,343(78.8)$ & $214(72.3)$ & $6.920(0.009)$ \\
\hline Not improved & $899(21.2)$ & $82(27.7)$ & \\
\hline \multicolumn{4}{|l|}{ Post-discharge } \\
\hline Home & $2,895(68.0)$ & $160(54.1)$ & $25.430(<0.001)$ \\
\hline Transfer & $727(17.1)$ & $75(25.3)$ & \\
\hline Decease & $620(14.6)$ & $61(20.6)$ & \\
\hline \multicolumn{4}{|l|}{ Hospital area } \\
\hline Capital city & $863(20.3)$ & $52(17.6)$ & $1.530(0.465)$ \\
\hline Metropolitan-city & $1,490(35.1)$ & $104(35.1)$ & \\
\hline Provincial area & $1,889(44.5)$ & $140(47.3)$ & \\
\hline Inpatient period & $32.55(44.37)$ & $42.65(53.21)$ & $-3.190(0.002)$ \\
\hline
\end{tabular}

Values are presented as $\mathrm{n}(\%)$ or mean (SD). CCI: Charlson Comorbidity Index. 
significant differences. Patients receiving medical aid were older than the ones with national health insurance, and there was higher proportion of patients with a severity level of 1 or higher. In the treatment outcome, the not-improved, transferred, and deceased cases after discharge were higher, but there was no statistically significant difference in the loca-

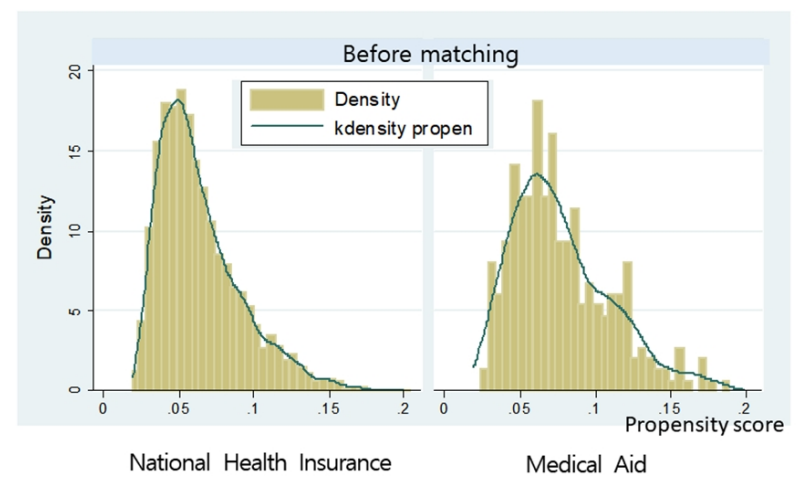

tion of the medical facilities. The inpatient period for the patients receiving medical aid was 42.65 days, which was significantly longer than that of the patients with national health insurance ( 32.55 days) (Table 1$)$.

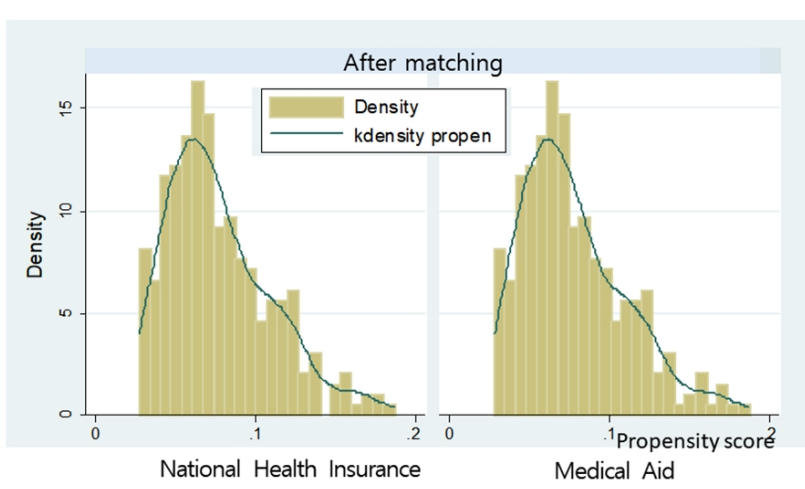

Figure 1. Comparison of propensity score distribution. X-axis: propensity score, y-axis: density.

Table 2. General characteristics after matching

\begin{tabular}{|c|c|c|c|}
\hline Characteristic & National health insurance $(n=296)$ & Medical Aid $(n=296)$ & $\chi^{2} / t(p)$ \\
\hline \multicolumn{4}{|l|}{ Sex } \\
\hline Male & $127(42.9)$ & $127(42.9)$ & $1.000(1.000)$ \\
\hline Female & $169(57.1)$ & $169(57.1)$ & \\
\hline Age (y) & $63.58(13.10)$ & $63.03(14.80)$ & $0.470(0.637)$ \\
\hline \multicolumn{4}{|l|}{ Residence } \\
\hline Consistency & $211(71.3)$ & $210(71.0)$ & $0.010(0.928)$ \\
\hline Inconsistency & $85(28.7)$ & $86(29.1)$ & \\
\hline \multicolumn{4}{|l|}{ Charlson Index } \\
\hline CCI 0 & $133(44.9)$ & $128(43.2)$ & $1.450(0.694)$ \\
\hline CCI 1 & $100(33.8)$ & $99(33.5)$ & \\
\hline CCI 2 & $34(11.5)$ & $31(10.5)$ & \\
\hline CCI $3+$ & $29(9.8)$ & $38(12.8)$ & \\
\hline \multicolumn{4}{|l|}{ Hospital admission } \\
\hline Emergency & $274(92.6)$ & $262(88.5)$ & $2.840(0.092)$ \\
\hline Outpatient & $22(7.4)$ & $34(11.5)$ & \\
\hline \multicolumn{4}{|l|}{ Treatment outcome } \\
\hline Improved & $211(71.3)$ & $214(72.3)$ & $0.080(0.784)$ \\
\hline Not improved & $85(28.7)$ & $82(27.7)$ & \\
\hline \multicolumn{4}{|l|}{ Post-discharge } \\
\hline Home & $157(53.0)$ & $160(54.1)$ & $0.070(0.967)$ \\
\hline Transfer & $76(25.7)$ & $75(25.3)$ & \\
\hline Decease & $63(21.3)$ & $61(20.6)$ & \\
\hline \multicolumn{4}{|l|}{ Hospital area } \\
\hline Capital city & $46(15.5)$ & $52(17.6)$ & $0.540(0.765)$ \\
\hline Metropolitan-city & $110(37.2)$ & $104(35.1)$ & \\
\hline Provincial area & $140(47.3)$ & $140(47.3)$ & \\
\hline Inpatient period & $30.27(38.75)$ & $42.65(53.21)$ & $-3.240(0.006)$ \\
\hline
\end{tabular}

Values are presented as $\mathrm{n}(\%)$ or mean (SD).

CCI: Charlson Comorbidity Index. 
Propensity score matching of medical aid patients and national health insurance patients

Figure 1 shows the analysis of pre-post matching scores of patients with medical aid patients and those with national health insurance. The score distribution showed to be similar after matching. It was confirmed that the variables of the two groups were similarly matched.

\section{Characteristic analysis after PSM matching}

Table 2 shows $100 \%$ matching of 296 patients with medical aid as a result of 1:1 matching by the PSM. The variables that showed significant differences before matching showed no statistically significant difference after matching. After PSM, the duration of hospitalization for patients with medi- cal aid was 42.65 days, which was significantly longer than 30.27 days of the patients with national health insurance.

Factors affecting the inpatient period for hemorrhagic stroke patients

Table 3 shows the results of regression analysis on the inpatient period before and after PSM as dependent variables. The variables that were statistically significant both before and after PSM were age, health insurance, severity level, hospital admission process, and treatment outcome. However, post-discharge status and place of location were not significant after PSM.

Factors affecting the inpatient period were older age, admission to outpatient clinics, no improvement in treatment outcome, and the decease state, which shortened the in-

Table 3. Regression analysis on the factors affecting inpatient period

\begin{tabular}{|c|c|c|c|c|}
\hline \multirow{2}{*}{ Characterstic } & \multicolumn{2}{|c|}{ Pre-PSM } & \multicolumn{2}{|c|}{ Post-PSM } \\
\hline & $\beta$ & $\mathrm{t}(p)$ & $\beta$ & $\mathrm{t}(p)$ \\
\hline \multicolumn{5}{|l|}{ Sex } \\
\hline Male & 1.000 & & 1.000 & \\
\hline Female & 0.047 & $1.850(0.065)$ & 0.010 & $0.120(0.902)$ \\
\hline Age (y) & -0.003 & $-3.270(0.001)$ & -0.007 & $-2.440(0.015)$ \\
\hline \multicolumn{5}{|l|}{ Health care } \\
\hline National health insurance & 1.000 & & 1.000 & \\
\hline Medical aid & 0.220 & $4.310(<0.001)$ & 0.264 & $3.510(<0.001)$ \\
\hline \multicolumn{5}{|l|}{ Residence } \\
\hline Consistency & 1.000 & & 1.000 & \\
\hline Inconsistency & -0.056 & $-1.870(0.061)$ & -0.168 & $-1.900(0.058)$ \\
\hline \multicolumn{5}{|l|}{ Charlson Index } \\
\hline CCI 0 & 1.000 & & 1.000 & \\
\hline CCI 1 & 0.127 & $4.350(<0.001)$ & 0.214 & $2.450(0.014)$ \\
\hline CCI 2 & 0.432 & $9.660(<0.001)$ & 0.350 & $2.760(0.006)$ \\
\hline CCI $3+$ & 0.716 & $15.190(<0.001)$ & 0.642 & $5.050(<0.001)$ \\
\hline \multicolumn{5}{|l|}{ Hospital admission } \\
\hline Emergency & 1.000 & & 1.000 & \\
\hline Outpatient & -0.483 & $-13.130(<0.001)$ & -0.359 & $-2.710(0.007)$ \\
\hline \multicolumn{5}{|l|}{ Treatment outcome } \\
\hline Improved & 1.000 & & 1.000 & \\
\hline Not improved & -0.967 & $-19.070(<0.001)$ & -0.727 & $-5.250(<0.001)$ \\
\hline \multicolumn{5}{|l|}{ Post-discharge } \\
\hline Home & 1.000 & & 1.000 & \\
\hline Transfer & 0.228 & $6.260(<0.001)$ & 0.144 & $1.490(0.137)$ \\
\hline Decease & 0.064 & $1.080(0.280)$ & 0.033 & $0.200(0.838)$ \\
\hline \multicolumn{5}{|l|}{ Hospital area } \\
\hline Capital city & 1.000 & & 1.000 & \\
\hline Metropolitan-city & -0.122 & $-3.420(0.001)$ & -0.062 & $-0.530(0.595)$ \\
\hline Provincial area & -0.148 & $-4.320(<0.001)$ & -0.177 & $-1.600(0.111)$ \\
\hline
\end{tabular}

PSM: propensity score matching. 
patient period. Inpatient period was found to be longer in the patients with medical aid and higher severity level.

\section{Discussion}

The purpose of this study was to compare the inpatient periods of hemorrhagic stroke suvivors with national health insurance and medical aid by using the PSM analysis. In this study, we proposed a methodology to calculate medical costs and assess hospital performance [19]. Furthermore, the resource utilization measurement variable that indirectly diagnoses medical service outcome and qualitative variation is the index that should be managed because of the importance for hospital administration and medical policy with high forecasting power.

The results of this study showed that post-stroke survivors with medical aid were older, more severe, had less improvement, and were more likely to be transferred, depleted, or deceased than the persons with national health insurance. The inpatient period of patients with medical aid was significantly longer than that of those with national health insurance, and the difference between the two groups after the PSM was increased by 2.28 days.

The length of stay in hospital of hemorrhagic stroke survivors in this research is longer than survivors in the Netherlands, which is 25.8 days [15], 6-8 days in the USA [20], and 10.6 days in Germany [21]. As such, the variations in the length of hospital of each country is different depending on the time of the stroke attack, the medical system, method of payment to medical insurance and effects of medical service payments system on subjects. The study that matched the type of health care by PSM reported similar results to this study in which the patients with medical aid used medical services more often than the ones with national health insurance.

Patients with medical aid have a relatively higher incidence of improper hospital stay and re-admissions compared to the ones with national health insurance. Patients with national health insurance had longer re-admission periods and higher total medical expenses, but their daily medical costs were lower [20,22]. The incidence of stroke is higher in males than in females, but the life expectancy of females is higher, resulting in a higher rate of post-stroke complications, and comorbidities, such as hypertension and cardiovascular diseases increases the difficulties in the management and treatment process of stroke [23]. The type of health care is a factor affecting the length of hospital stay for hemorrhagic stroke survivors. Systematic and comprehensive governmental management for the transfer of patients with medical aid to long-term care facilities under the National Health Insurance System and the Long-term Care Insurance System for the Elderly can reduce unnecessary hospitalization and re-admissions to acute care medical institutions.

The limitation of this study was that it did not fully include various factors affecting the inpatient period, especially limiting the supplier factors only to hospitals. A comprehensive comparative study that reflects the patient's personal characteristics, family support, and other alternative services in the community in future studies may provide a clearer picture of the differences in the inpatient period. However, this study differs from the existing papers in that it analyzed the differences of the inpatient period according to the type of health care in hemorrhagic stroke survivors only, not the overall patient population. It is significant that the PSM is used to clarify the differences in inpatient periods by controlling the influence factors other than the type of health care. The results of this study may be used to provide guidelines in the decision making process for efficient utilization of medical resources.

In conclusion, this study compared the inpatient periods of hemorrhagic stroke survivors with national health insurance and medical aid benefits by using the PSM analysis. After matching, the factors influencing the inpatient period were found to be treatment outcome, severity of disease, hospital admission process, and type of health care. The inpatient period was found to be longer in the patients receiving medical aid and with higher severity level of disease. The findings of this study can be used in developing a systematic and efficient care system for patients who use longterm care facilities.

\section{Acknowledgements}

This research was supported by the Ministry of Science and ICT (MSIT), Korea, under the Information Technology Research Center (ITRC) support program (IITP-2019-20141-00720) supervised by the Institute for Information \& communications Technology Planning \& Evaluation (IITP) and was also supported by the Bio \& Medical Technology Development Program of the National Research Foundation (NRF) funded by the Ministry of Science, ICT \& Future Planning (NRF-2015M3A9D7067388). 


\section{Conflict of Interest}

The authors declared no potential conflicts of interest with respect to the authorship and/or publication of this article.

\section{References}

1. OECD. Health at a Glance 2015: OECD indicators. Paris: OECD Publishing; 2015.

2. Ministry of Health and Welfare. Survey of the elderly [Internet]. Sejong: Ministry of Health and Welfare, 2018. [cited 2019 Mar 18] Available from: http://meta.narastat.kr/metasvc/index.do? confmNo=117071.

3. Lee SG, Jeon SY. The relations of socioeconomic status to health status, health behaviors in the elderly. J Prev Med Public Health 2005;38:154-62.

4. Statistics Korea. The prevalence rate (diagnosis criteria) and current treatment rate by sex of the elderly [Internet]. Daejeon: Statistics Korea, 2018. [cited 2019 Mar 18] Available from: http://kosis.kr/statHtml/statHtml.do?orgId=117\&tblId=DT_ 117071_018\&vw_cd=MT_ZTITLE\&list_id=117_11771_003_ 117_11771_003_06.

5. Russo CA, Andrews RM. Hospital stays for stroke and other cerebrovascular diseases, 2005: statistical brief \#51. Healthcare Cost and Utilization Project (HCUP) statistical briefs. Rockville (MD): Agency for Healthcare Research and Quality (US); 2006.

6. Kim SM, Hwang SW, Oh EH, Kang JK. Determinants of the length of stay in stroke patients. Osong Public Health Res Perspect 2013;4:329-41.

7. Lim JH, Cheon SH. Analysis of variation in length of stay (LOS) after ischemic and hemorrhagic stroke using the Charlson Comorbidity Index (CCI). J Phys Ther Sci 2015;27:799-803.

8. Kang SH, Kim WJ, Seok HS. The variation of factors of severity-adjusted length of stay (LOS) in acute stroke patients. J Digit Policy Manag 2013;11:221-33.

9. Lim SJ, Kim HJ, Nam CM, Chang HS, Jang YH, Kim S, et al. Socioeconomic costs of stroke in Korea: estimated from the Korea national health insurance claims database. J Prev Med Public Health 2009;42:251-60.

10. Kim YH, Moon JW, Kim KH. The determinant factors and medical charges pattern by length of stay in hospital. Korean J Hosp Manag 2010;15:15-26.

11. Freitas A, Silva-Costa T, Lopes F, Garcia-Lema I, Teixeira-Pinto
A, Brazdil P, et al. Factors influencing hospital high length of stay outliers. BMC Health Serv Res 2012;12:265.

12. Youn KI. Comparisons of health care utilization patterns and outcome for national health insurance and medical aid program cancer patients. J Korea Soc Health Inform Stat 2014;39:42-59.

13. Joo JM, Kwon SM. Difference in outpatient medical expenditure and physician practice patterns between medicaid and health insurance patients. Health Policy Manag 2009;19:125-41.

14. Kwon YD, Jang HJ, Choi YJ, Yoon SS. Nationwide trends in stroke hospitalization over the past decade. J Korean Med Assoc 2012;55:1014-25.

15. Evers S, Voss G, Nieman F, Ament A, Groot T, Lodder J, et al. Predicting the cost of hospital stay for stroke patients: the use of diagnosis related groups. Health Policy 2002;61:21-42.

16. Seo HJ, Yoon SJ, Lee SI, Lee KS, Yun YH, Kim EJ, et al. A comparison of the Charlson comorbidity index derived from medical records and claims data from patients undergoing lung cancer surgery in Korea: a population-based investigation. BMC Health Serv Res 2010;10:236.

17. Quan H, Li B, Couris CM, Fushimi K, Graham P, Hider P, et al. Updating and validating the Charlson comorbidity index and score for risk adjustment in hospital discharge abstracts using data from 6 countries. Am J Epidemiol 2011;173:676-82.

18. Goldstein LB, Samsa GP, Matchar DB, Horner RD. Charlson Index comorbidity adjustment for ischemic stroke outcome studies. Stroke 2004;35:1941-5.

19. Suh HS, Kang HY, Kim J, Shin E. Effect of health insurance type on health care utilization in patients with hypertension: a national health insurance database study in Korea. BMC Health Serv Res 2014; 14:570.

20. Diringer MN, Edwards DF, Mattson DT, Akins PT, Sheedy CW, Hsu CY, et al. Predictors of acute hospital costs for treatment of ischemic stroke in an academic center. Stroke 1999;30:724-8.

21. Heuschmann PU, Kolominsky-Rabas PL, Misselwitz B, Hermanek P, Leffmann C, Janzen RW, et al. Predictors of in-hospital mortality and attributable risks of death after ischemic stroke: the German Stroke Registers Study Group. Arch Intern Med 2004; 164:1761-8.

22. Reeves MJ, Bushnell CD, Howard G, Gargano JW, Duncan PW, Lynch G, et al. Sex differences in stroke: epidemiology, clinical presentation, medical care, and outcomes. Lancet Neurol 2008; 7:915-26.

23. Bae HJ, Kim HS, Lee KS. Utilization of health care resources and costs of stroke patients: patients' perspective. J Korean Neurol Assoc 2004;22:583-9. 